\title{
A Fuzzy Controlled Incremental Conductance Method for Z-Source PV Inverter MPPT
}

\author{
Jingwei Zhang ${ }^{1}$, Honghua Wang ${ }^{1, *}$, Chengliang Wang ${ }^{2}$, Wei Han ${ }^{1}$, Zhebei Wang ${ }^{1}$ and Rong Sun ${ }^{3}$ \\ ${ }^{1}$ College of Energy and Electrical Engineering, Hohai University, Nanjing 211100, Jiangsu Province, China \\ ${ }^{2}$ Jiangsu Frontier Electric Technology Co., Ltd., Nanjing 211100, Jiangsu Province, China \\ ${ }^{3}$ Jiangsu Electric Power Company Research Institute, Nanjing 211100, Jiangsu Province, China \\ *Corresponding author
}

\begin{abstract}
Based on conventional incremental conductance (IncCond) method, a fuzzy controlled incremental conductance controller is proposed for $\mathrm{Z}$-source $\mathrm{PV}$ inverter to realize its maximum power point tracking (MPPT). The principle of $Z$ source PV inverter is illustrated. Then an improvement of SVPWM for Z-source inverter is introduced for DC-AC conversion. The fuzzy rules and membership functions are designed to generate adaptive voltage step size of incremental conductance. Simulation and experiment results reveal that proposed fuzzy controlled Inc-Cond method can track maximum power point (MPP) much faster and achieve less fluctuation at the vicinity of MPP. Experimental results also verified proposed fuzzy algorithm is feasible to realize with DSP controllers, and the improved SVPWM combined with proposed algorithm can be easily implemented with Z-source PV inverter.
\end{abstract}

Keywords-MPPT; Z-source inverter; photovoltaic; fuzzy control; incremental conductance

\section{INTRODUCTION}

In recent decades, radiation energy capture is always a hot research issue during the application of photovoltaic system. A high efficiency method for maximum power point tracking (MPPT) can assist PV system generate much more power. The conventional PV system utilized boost converter to trace maximum power and an inverter to provide $\mathrm{AC}$ power for loads. These two stages DC-AC converters have more power devices, which increase energy loss. The efficiency of whole system is decreased inevitably and system structure is complicated [1]. Then F. Z. Peng et al. proposed Z-source inverters [2,3], which utilizes shoot-through duty of inverter bridge to boost output voltage of Z-source network, and the inverter current can also be controlled independently. Hence it has higher efficiency and stability.

Based on Z-source inverter and conventional space vector pulse width modulation (SVPWM), this paper proposed the fuzzy controlled incremental conductance for MPPT. Firstly, the Z-source inverter is introduced and conventional SVPWM is improved to generate shoot-through duty for Z-source. Then the fuzzy controller is illustrated and combined with traditional incremental conductance. Simulation and experiments are implemented to verify feasibility of proposed algorithm.

\section{CONTROL OF Z-SOURCE INVERTER AND IMPROVEMENT OF SVPWM}

\section{A. Principle of Z-source PV Inverter}

Figure 1 reveals the conventional Z-source network. It is formed by inductors and capacities which connect in X-type. The principle of boost voltage can be expressed by (1) $[2,3]$ :

$$
\left\{\begin{array}{l}
U_{C}=\frac{1-D_{0}}{1-2 D_{0}} U_{P V} \\
U_{Z}=\frac{1}{1-2 D_{0}} U_{P V}=B U_{P V}
\end{array}\right.
$$

$U_{P V}$ denotes output voltage of PV array, $U_{C}$ represents the voltage of capacitance in $Z$-source network, $U_{Z}$ is output voltage of $Z$-source network, $D_{0}$ depicts the shoot-through duty, $B$ is the boost factor of $Z$-source network. Equation (1) indicates $U_{Z}$ can be boosted via adjusting shoot-through duty in the range of $[0,0.5)$, and the range of boost factor $B$ is $[1, \infty)$ theoretically. Hence the voltage gain of Z-source inverter is:

$$
G=\frac{U_{A C_{-} \text {peak }}}{U_{P V}}=\frac{\frac{M}{2} U_{Z}}{U_{P V}}=\frac{M B}{2}
$$

where $G$ is voltage gain, $M$ is modulation index of inverter.

Assuming system load is constant, for instance, threephase AC loads are consist of constant resistance. Then the generated power of PV array can be regulated via controlling voltage of PV array, which is tracing the maximum power point. For a Z-source inverter, voltage of PV array can be controlled by adjusting shoot-through duty of inverter. In this paper, the SVPWM is improved for inserting shoot-through time during modulation.

\section{B. Improvement of SVPWM Control}

SVPWM is commonly applied in modulating ordinary three-phase inverter. In order to avoid DC-link shoot-through, the dead-time is necessary during driving IGBTs. However, 
different from conventional inverters, as mentioned above, Zsource inverter need additional shoot-through time. A convenient method for shoot-through time insertion is utilized in this paper.

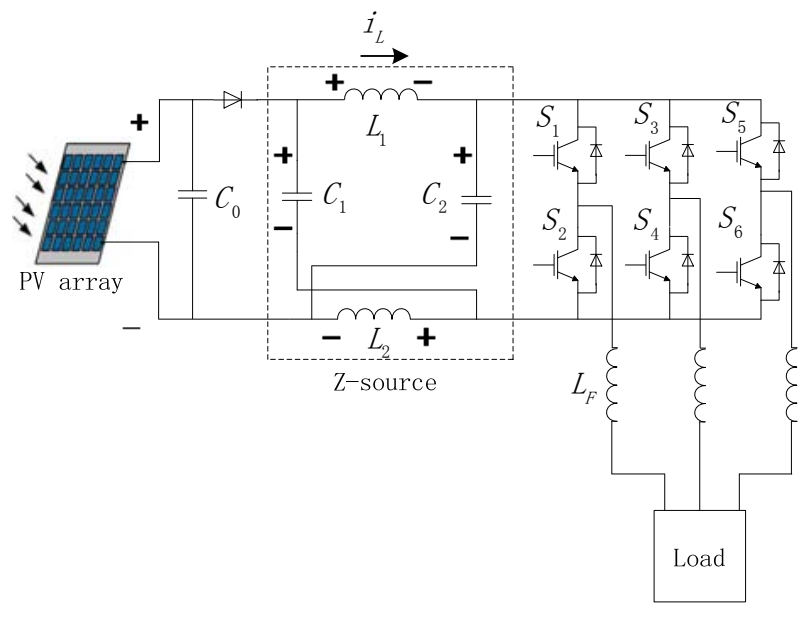

FIGURE I. STRUCTURE OF Z-SOURCE PV INVERTER.

In the inverter bridge shown in Figure 1, the on-state of IGBT on the upper leg is denoted by ' 1 ', and the on-state of corresponding device on the lower leg is ' 0 '. Then space vectors can be depicted as 6 effective vectors, $V_{1}$ to $V_{6}$, and 2 zero vectors, $V_{0}$ and $V_{7}$. They are both non-shoot-through vectors, as shown in Figure 2.

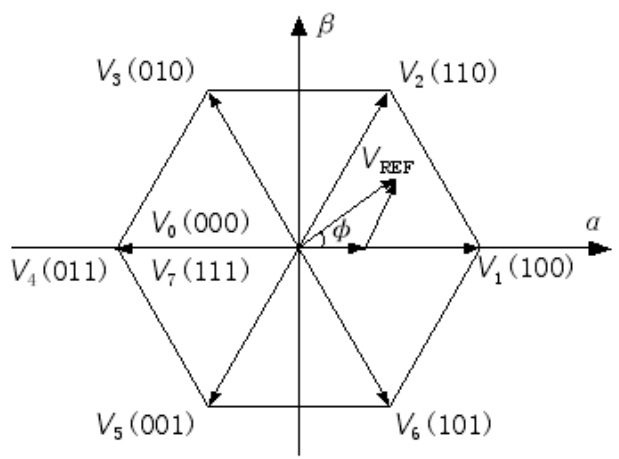

\section{FIGURE II. DISTRIBUTION OF VOLTAGE VECTORS ON THE} COMPLEX PLANE.

The plane is divided into sectors by six effective vectors. Assuming the required voltage vector $V_{R E F}$ locates in the first sector, which is delimited between vector $V_{1}$ and $V_{2}$, so $V_{R E F}$ can be calculated as:

$$
V_{R E F}=\frac{T_{1}}{T_{S}} V_{1}+\frac{T_{2}}{T_{S}} V_{2}+\frac{T_{0,7}}{T_{S}} V_{0}
$$

where $T_{1}, T_{2}$ and $T_{0,7}$ are effective time of vector $V_{1}, V_{2}$ and $V_{0}$ or $V_{7} ; T_{S}$ is time of a switching period. Hence (4) can be deduced:

$$
T_{S}=T_{1}+T_{2}+T_{0}+T_{7}
$$

Due to the similarity, the principle for calculating vector $V_{R E F}$ in other 5 sectors are omitted.

Based on SVPWM mentioned above, shoot-through vector is introduced. Firstly, symmetric 7 non-shoot-through vectors are used in a switching period $T_{S}$. Then shoot-through time is inserted into transition of two adjacent vectors $[3,4]$. In sector I, for instance, the conducting sequences of 6 IGBTs $S_{1}$ to $S_{6}$, are shown in Figure 3. Shaded areas represent working period of shoot-through time $T_{S T}$, and it is divided into 6 parts in one switching period. It should be pointed out that this insertion method can reduce inverter harmonics and is applicable in micro controller, e.g. MCUs, DSPs.

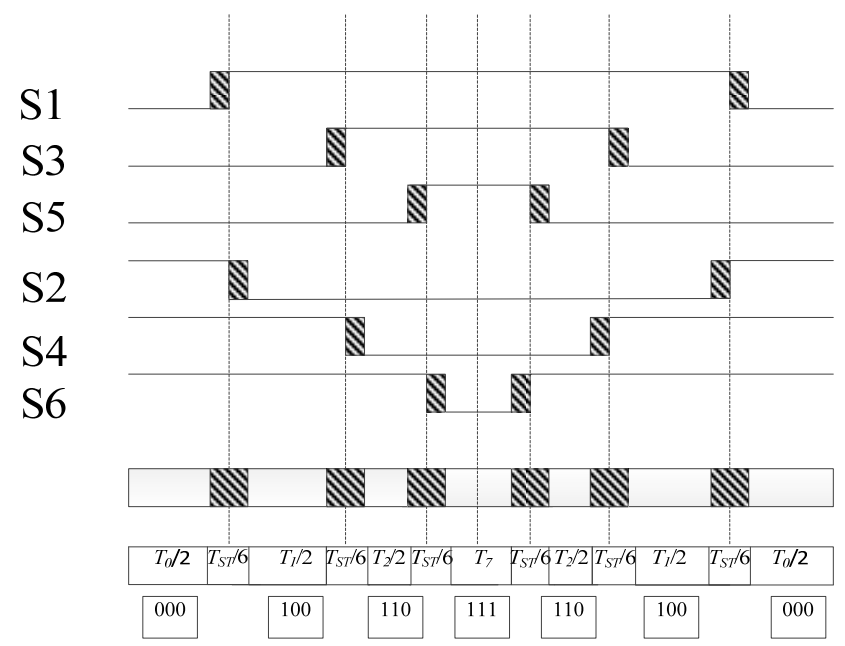

FIGURE III. SVPWM SWITCHING SIGNALS IN SECTOR I.

Thus, (4) should be modified as follows:

$$
T_{S}=T_{1}+T_{2}+T_{0}+T_{7}+T_{S T}
$$

The conducting sequences in other 5 sectors are omitted due to the similarity.

\section{DESIGN OF FUZZY CONTROLLED INCREMENTAL \\ CONDUCTANCE}

The output characteristics of PV panel is influenced by ambient irradiance and temperature significantly. Its nonlinear feature requires higher performance of controller. Commonly, MPPT methods include: fractional open-circuit voltage method, perturb and observe $(\mathrm{P} \& \mathrm{O})$, incremental conductance (Inc-Cond), et al [5]. Fuzzy logic based MPPT techniques also have been applied in recent years. It uses error and derivative of error as controller input and output duty ratio to realize MPPT [5,6]. In this paper, different from conventional fuzzy logic controlled MPPT, a fuzzy controller is utilized to generate adaptive voltage step size for incremental conductance to achieve faster tracking speed and better dynamic performance, as Figure 4 describes. 


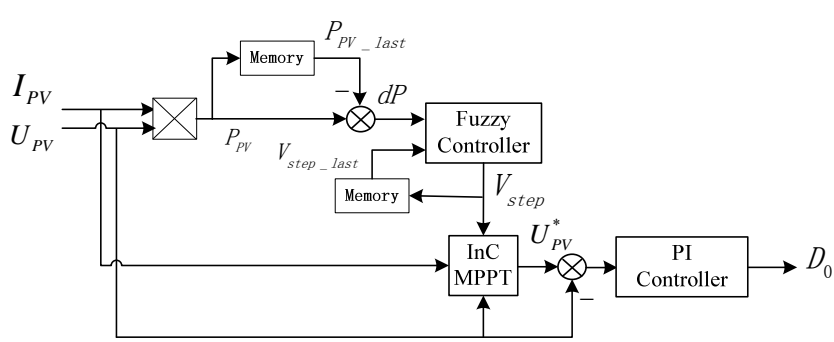

FIGURE IV. CONTROL DIAGRAM OF PROPOSED FUZZY CONTROLLED INCREMENTAL CONDUCTANCE

$d P$ is power deviation in last two sampling period. $V_{\text {step_last }}$ is the last output of fuzzy controller, $V_{\text {step }}$ is controller output in current sampling period. Hence, the input of fuzzy controller are $d P$ and $V_{\text {step_last, }}$, then voltage step size, $V_{\text {step }}$ is determined by fuzzy controller. Due to input parameters of controller are actual physical variables, fuzzy rules are much easier to design. Controller inputs $d P$ and $V_{\text {step last }}$ and output $V_{\text {step }}$ are both divided into 6 fuzzy sets, i.e. negtive big (NB), negative medium (NM), negative small (NS), positive small (PS), positive medium (PM) and positive big (PB). Considering the power-voltage $(\mathrm{P}-\mathrm{V})$ characteristics of $\mathrm{PV}$ array, fuzzy rules are designed, as shown in Table I. It should be pointed out that designed fuzzy rules table is not a strictly symmetrical array. The reason is P-V curve of PV array is asymmetric, so different voltage steps should be chosen at different sides of maximum power point (MPP). In fact, if current operating point is on the left side of MPP, a large step should be used to accelerate searching speed, however, if current operating point is on the right side of MPP, a small voltage step is necessary to avoid over-swing at the vicinity of the MPP.

TABLE I. DESIGNED FUZZY RULES TABLE

\begin{tabular}{|c|c|c|c|c|c|c|}
\hline \multirow{2}{*}{$\boldsymbol{d P}$} & \multicolumn{6}{|c|}{$\boldsymbol{V}_{\text {ste__last }}$} \\
\cline { 2 - 7 } & NB & NM & NS & PS & PM & PB \\
\hline NB & PB & PM & PS & NS & NM & NM \\
\hline NM & PM & PS & PS & NS & NS & NM \\
\hline NS & PM & PS & PS & NS & NS & NM \\
\hline PS & NM & NS & NS & PS & PS & PM \\
\hline PM & NM & NS & NS & PS & PS & PM \\
\hline PB & NM & NS & NS & PS & PM & PB \\
\hline
\end{tabular}

Membership functions of inputs $d P, V_{\text {step last }}$ and output $V_{\text {step }}$ are also defined, as shown in Figure 5 (a) and (b). Same triangular membership functions are applied for $V_{\text {step_last }}$ and $V_{\text {step }}$ in the range of $[-6,6]$, and the range of $d P$ is $[-10,10]$. If input value is close to zero, corresponding triangular function is more abrupt to reduce controller output, and finally reduce output oscillation near MPP. Then the commonly used centroid method is applied for defuzzification.

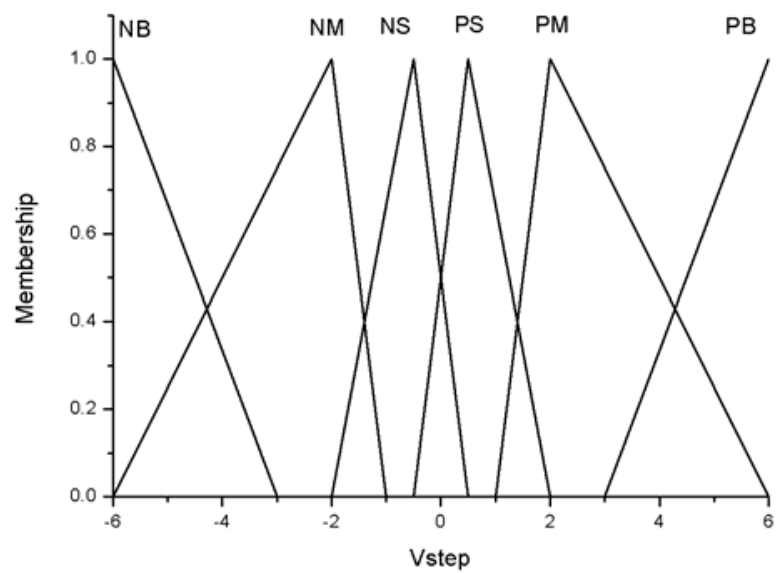

(a)

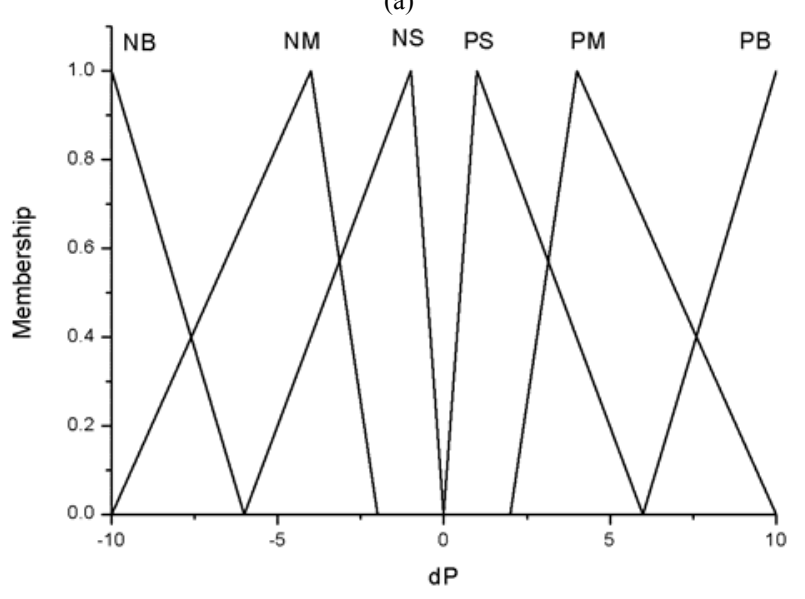

(b)

FIGURE V. MEMBERSHIP FUNCTIONS: (A) INPUT VSTEP LAST AND OUTPUT VSTEP, (B) INPUT DP

\section{SIMULATION AND EXPERIMENTS}

In order to verify the feasibility of proposed fuzzy controlled incremental conductance method for Z-source inverter, simulation and experiments are implemented. Firstly, a $50 \mathrm{~W}$ PV module is used to generate power. Its electrical characteristics under standard test condition (STC) are shown in Table II. Luft's model is applied to model the PV module in MATLAB/Simulink [7]. Considering voltage and current ripple of capacitors and inductors in Z-source, parameters of Z-source in Figure 1 are designed as: $L_{1}=L_{2}=1 \mathrm{mH}$, $C_{1}=C_{2}=330 \mathrm{uF}$. Modulation index of SVPWM is set as 0.6 , irradiance is $1 \mathrm{~kW} / \mathrm{m} 2$ and temperature is $25^{\circ} \mathrm{C}$ during simulating MPPT. Figure 6 gives the result of simulated voltage of PV module when system is stable. it fluctuates at the vicinity of $18.4 \mathrm{~V}$, which agrees with electrical characteristics in Table II. Figure 7 reveals the searching path during MPPT. The range of given reference voltage is set from $12 \mathrm{~V}$ to $21 \mathrm{~V}$, hence it rises from $12 \mathrm{~V}$ since system start-up. As Figure 8 shows that proposed algorithm applies larger voltage step to approach MPP then tighten the step when it is close to MPP, which agrees with ideas during designing fuzzy rules, and eventually operating point locates at the vicinity of MPP. 
TABLE II. ELECTRICAL CHARACTERISTICS OF PV MODULE

\begin{tabular}{|c|c|}
\hline Parameters & Value \\
\hline Power & $50 \mathrm{~W}$ \\
\hline Open circuit voltage, $\mathrm{V}_{\mathrm{OC}}$ & $21.5 \mathrm{~V}$ \\
\hline Short circuit current, $\mathrm{I}_{\mathrm{SC}}$ & $2.95 \mathrm{~A}$ \\
\hline Voltage at MPP, $\mathrm{V}_{\mathrm{m}}$ & $18.4 \mathrm{~V}$ \\
\hline Current at MPP, $\mathrm{I}_{\mathrm{m}}$ & $2.72 \mathrm{~A}$ \\
\hline Test irradiance & $1000 \mathrm{~W} / \mathrm{m}^{2}$ \\
\hline Test temperature & $25^{\circ} \mathrm{C}$ \\
\hline
\end{tabular}

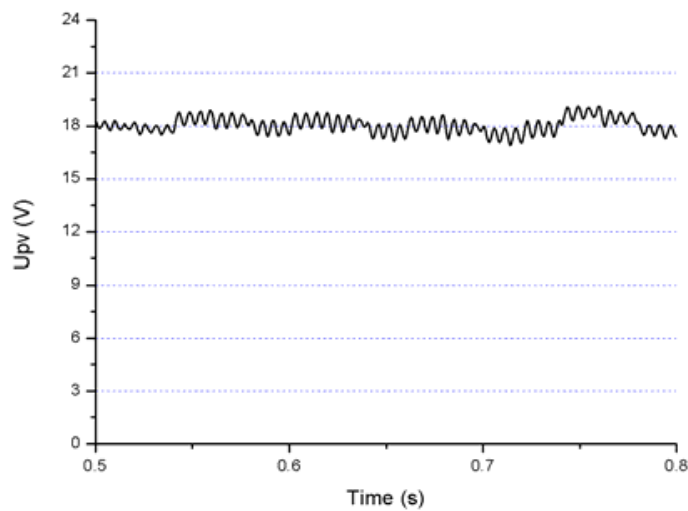

FIGURE VI. VOLTAGE CURVE OF PV MODULE

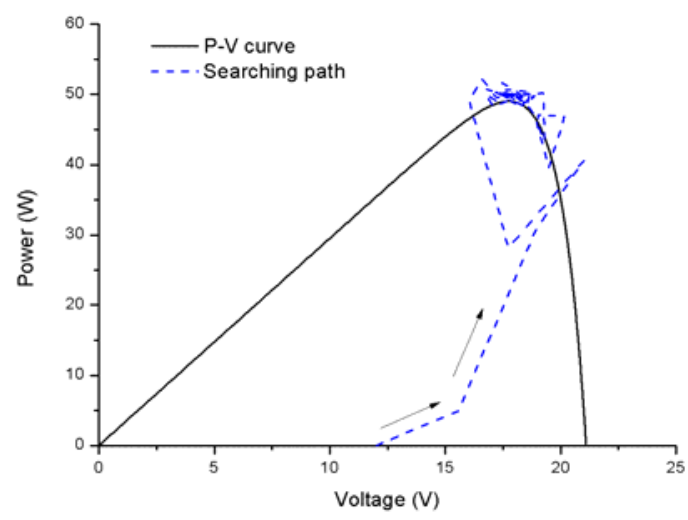

FIGURE VII. SEARCHING PATH OF PROPOSED FUZZY CONTROLLED INCREMENTAL CONDUCTANCE

Then, an experiment is carried out to verify feasibility of improved SVPWM. Circuit parameters in experiments conform with that in simulation. DSP TMS320F2812 is used as system controller. Power MOSFETs IRF3205 are used to consist the inverter bridge. 6 driver chips IR2110 are applied to drive each MOSFET independently to realize bridge shootthrough. Shoot-through duty is set as 0.24 during this experiment, and the switching frequency is $1 \mathrm{kHz}$. Figure 8 shows driving signals for inverter A phase bridge legs. In a switching period $1 \mathrm{~ms}$, shoot-through time in A phase is approximate 79us. Hence total shoot-through time in three phases is almost 237 us and agrees with pre-set. Duty deviation mainly comes from time cost during driving signals rising.

Another experiment is implemented for verifying proposed MPPT algorithm. In order to compare performance between conventional Inc-Cond and proposed fuzzy controlled IncCond, output voltage of PV module controlled by above two algorithms is focused, as Figure 9 reveals. The voltage step of conventional Inc-Cond is constant, $1 \mathrm{~V}$. The step size of proposed method converge to $0.6 \mathrm{~V}$. Figure 9 indicates proposed fuzzy controlled Inc-Cond MPPT method makes PV module voltage much more stable, which decreases energy lose during MPPT. Figure 10 reveals A phase voltage of inverter and its FFT result. The fundamental frequency is stable at $50 \mathrm{~Hz}$, corresponding root mean square(RMS) value is $3.71 \mathrm{~V}$. and low-order harmonics are approximate $0.15 \mathrm{~V}$. Hence, inverted voltage harmonics are less than $5 \%$, which verifies the applied improved SVPWM control for Z-source inverter is also feasible.

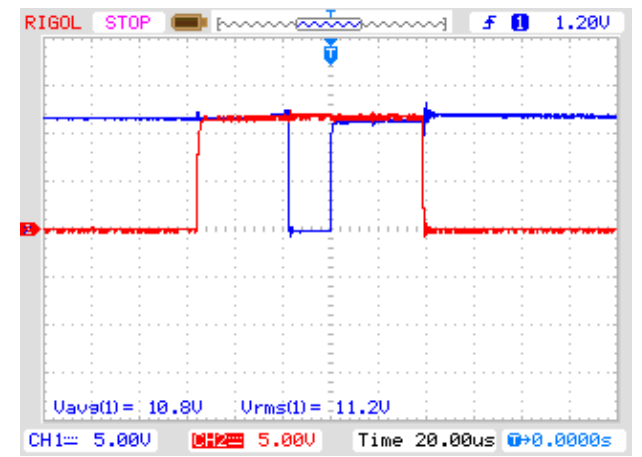

FIGURE VIII. DRIVING SIGNALS FOR MOSFETS IN A PHASE

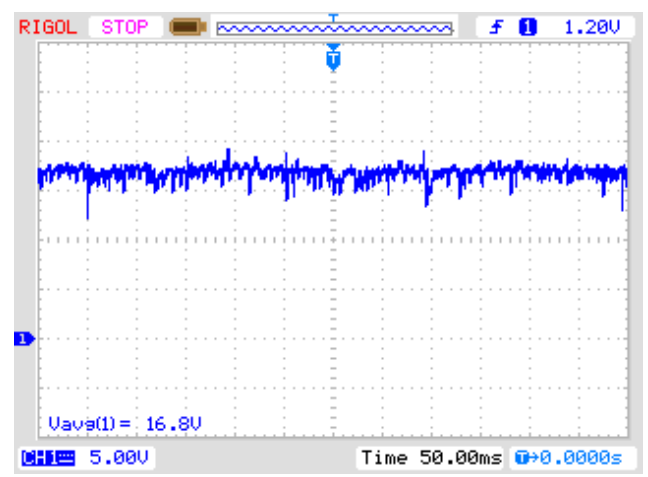

(a)

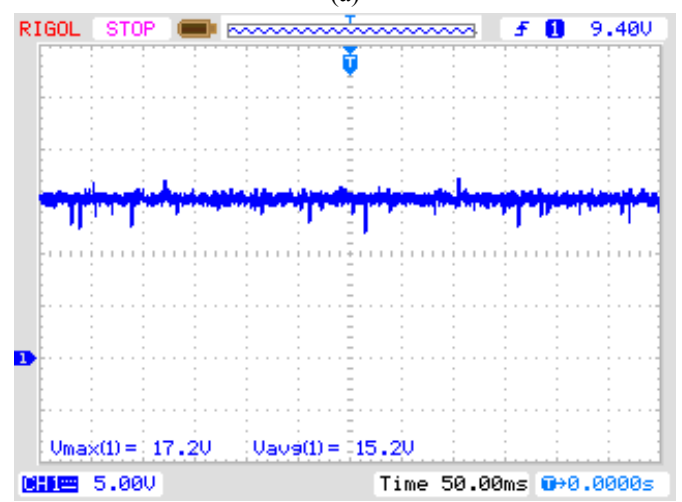

(b)

FIGURE IX. VOLTAGE CURVE OF PV MODULE UNDER DIFFERENT MPPT ALGORITHM: (A) RESULT OF CONVENTIONAL INC-COND MPPT, (B) RESULT OF PROPOSED FUZZY CONTROLLED INC-COND MPPT 


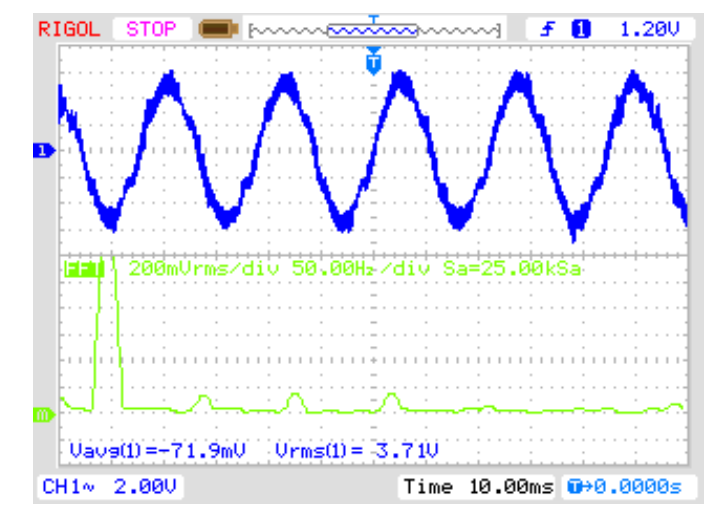

FIGURE X. A PHASE VOLTAGE CURVE AND FFT RESULT

\section{CONCLUSIONS}

The MPPT method based on fuzzy controlled incremental conductance is proposed in this paper. Different from conventional fuzzy PID control, proposed fuzzy inference is designed to infer voltage step size for incremental conductance. Corresponding simulation and experiments reveal that this method can trace MPP much faster, and more stable at the vicinity of MPP, and it can be combined with the improved SVPWM control for Z-source PV inverter.

\section{ACKNOWLEDGEMENT}

This research was supported by a grant from the State Grid Jiangsu electric power company project of China (No.J2014028).

\section{REFERENCES}

[1] S. B. Kjaer, J. K. Pederson, F. Blaabjerg, "A review of single-phase grid-connected inverters for photovoltaic modules," IEEE Trans. Industry Applications, vol. 41, no. 5, pp. 1292-1306, Sept. 2005.

[2] F. Z. Peng, A. Joseph, J. Wang, et al., "Z-source inverter for motor drives," IEEE Trans. Power Electronics, vol. 20, no. 4, pp. 857-863, Jul. 2005.

[3] K. Q. Qu, Q. Q. Niu, C. Yang, J. B. Zhao, "Battery Charge-Discharge Control Strategy Based on the Single Z-Source Three-Level SVPWM Inverter," presented at the 2013 IEEE Int. Conf. on Applied Superconductivity and Electromagnetic Devices (ASEMD), Beijing, China, Oct. 25-27, 2013.

[4] J. Ding, S. Xie, Y. Tang, et al. "Optimal design of the inductor in Zsource inverter with single phase shoot-through SVPWM strategy". presented at the 2010 IEEE Energy Conversion Congress and Exposition (ECCE),. Atlanta, GA, Sept. 12-16, 2010.

[5] B. Subudhi, R. Pradhan, "A comparative study on maximum power point tracking techniques for photovoltaic power system," IEEE Trans. Sustainable Energy, vol. 4, no. 1, pp. 89-98, Jan. 2013.

[6] S. Li, J. Bai, Q. Tang, L. Yuan, "A kind of improved control method for Z-source inverter MPPT," presented at the 201527 th Chinese Control and Decision Conference (CCDC), Qingdao, China, May 23-25, 2015.

[7] W. De Soto, "Improvement and validation of a model for photovoltaic array performance", M.S. thesis, Solar Energy Laboratory, Univ. of Wisconsin-Madison, Madison, WI, 2004. 